\title{
Structure of Aqueous Solutions of Acetonitrile Investigated by Acoustic and Positron Annihilation Measurements
}

\author{
K. Jerie, A. Baranowski \\ Institute of Experimental Physics, University of Wrocław \\ Borna 9, 50-205 Wrocław, Poland \\ S. KOZIOL \\ Waters Corp., 34 Maple St., Milford, MA 01757, USA \\ J. Gliński And A. Burakowski \\ Faculty of Chemistry, University of Wrocław, Poland
}

\begin{abstract}
We report the results of acoustic and positron annihilation measurements in aqueous solutions of acetonitrile $\left(\mathrm{CH}_{3} \mathrm{CN}\right)$. Hydrophobicity of the solute is discussed, as well as the possibility of describing the title system in terms of hydrophobic solvation. The concept of Levay et al. of calculating the "ideal" positronium lifetimes is applied, basing on the mean volume of cavities (holes) in liquid structure available for positronium pseudoatom. The same calculations performed using the Tao model of annihilation yield very different results. It can be concluded that either acetonitrile forms with water clathrate-like hydrates of untypical architecture, or it is too weak hydrophobic agent to form clathrate-like hydrates at all. The former interpretation seems to be more probable.
\end{abstract}

PACS numbers: 61.25.Em, 78.70.Bj

\section{Introduction}

Acetonitrile (further denoted as $\mathrm{ACN}$ ), $\mathrm{CH}_{3} \mathrm{CN}$, has extremely short hydrophobic part and, consequently, its hydrophobic or hydrophilic character of interactions with water is not clear. Below we present selected literature data showing how unclear it is. On the basis of comparative studies of many aqueous systems Matteoli and Lepori stated that the hydrophilic group of acetonitrile reduces its hydrophobicity almost as strong as the $\mathrm{OH}$ or $\mathrm{NH}_{2}$ groups do [1]. Let us note that the series of hydrophilic character of different groups proposed by 
Matteoli and Lepori is in contradiction with findings of Wakisaka et al. [2], while according to the former authors methanol and acetonitrile should be similar in hydrophobic character (methanol should be even weaker). The number of authors who are in favour of unquestionable hydrophobic character of interactions of ACN with water is, however, prevailing. Thus, it is not clear how acetonitrile is solvated by water and which is the kind of hydration. The aim of this paper is to apply two independent methods, sound velocity and positron annihilation, to find differences or similarities of the system water-acetonitrile with other aqueous systems where hydrophobic effects dominate. The model system used for such comparison will be water-ethanol [3].

\section{Experimental}

Chemicals. Acetonitrile, $\mathrm{CH}_{3} \mathrm{CN}$ (Baker, HPLC grade) was glass-distilled. Water was doubly distilled and tested for purity by conductivity. Solutions were prepared gravimetrically and stored in glass containers not longer than two weeks, without contacting with air.

Acoustic measurements. The ultrasonic velocity was measured with an accuracy of $\pm 0.1 \mathrm{~m} / \mathrm{s}$ using the pulse method (computer-steered apparatus OPKUD, Optel, Poland). Density was measured using a vibrating pipe method (apparatus Ecolab MG-2, Poland) with the accuracy better than $\pm 0.02 \mathrm{~kg} / \mathrm{m}^{3}$. Temperature was changed from 283 to $308 \mathrm{~K}$ with intervals of $2-3 \mathrm{~K}$. The results (temperature dependences of density and sound velocity) were approximated using linear functions and the values interpolated at chosen temperatures were further used for visualizations and interpretations. The adiabatic compressibility coefficients $\beta$ were calculated from the Laplace equation $\beta=\left(\rho c^{2}\right)^{-1}$ (where $\rho$ - density of the liquid, $c$ - sound velocity). The concentration dependences of the compressibility for the systems investigated are shown in Fig. 1.

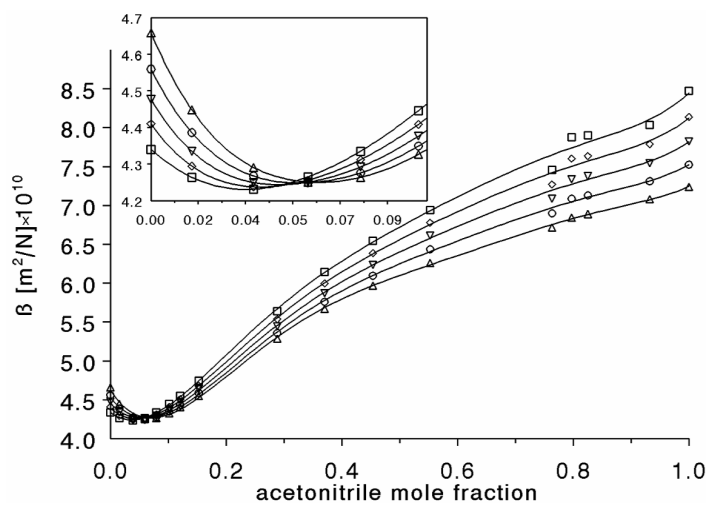

Fig. 1. Adiabatic compressibility coefficients of water + acetonitrile solutions plotted vs. ACN mole fraction. $\Delta-288.15 \mathrm{~K} ; \bigcirc-293.15 \mathrm{~K} ; \nabla-298.15 \mathrm{~K} ; \diamond-303.15 \mathrm{~K} ; \square$ - $308.15 \mathrm{~K}$. The region of low acetonitrile concentrations is shown in the inserted graph. 
Positron mean lifetimes. The annihilation measurements were carried out at room temperature (approximately $25^{\circ} \mathrm{C} \pm 2$ ) with a standard measuring device based on the "fast-slow" coincidence technique as described elsewhere [4]. The obtained spectra were resolved using POSITRONFIT program into three components with lifetimes $\tau_{1}, \tau_{2}, \tau_{3}$ and corresponding intensities $I_{1}, I_{2}, I_{3}$. Only the component with the longest lifetime (indexed as three) is of our interest here. Let us note the condition $I_{1}+I_{2}+I_{3}=100 \%$.

The concentration dependences of the long-lived component's parameters are shown in Figs. 2 and 3 (lifetime $\tau_{3}$ and intensity $I_{3}$, respectively). For comparison, the corresponding literature results obtained for the system water + ethanol [3] (where hydrophobic hydration dominates, i.e. quasicrystalline, clathrate-like hydrates are formed) are also shown in these figures.

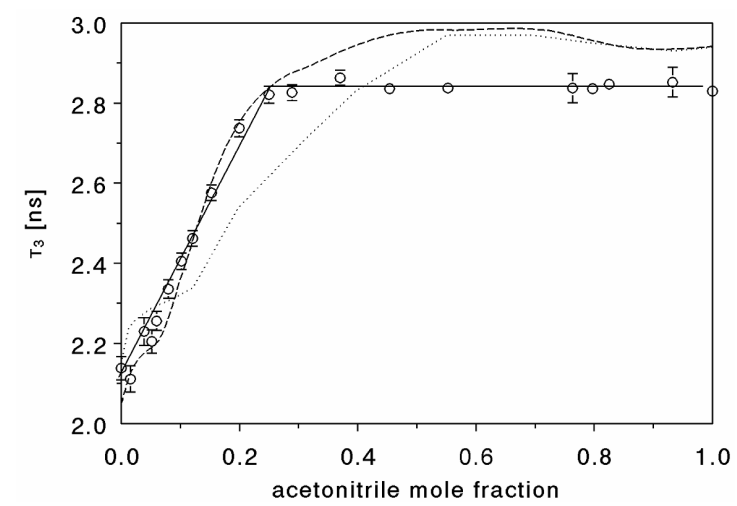

Fig. 2. Lifetimes of the long-lived component of the annihilation spectrum $\tau_{3}$ vs. ACN mole fraction in water. Dashed line - the results for water + ethanol mixtures [3], dotted line - those for water + methanol [13].

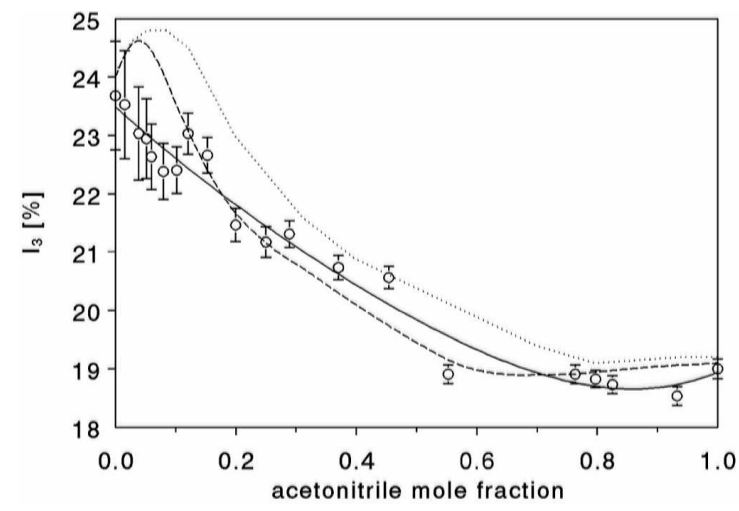

Fig. 3. Intensities of the long-lived component of the annihilation spectrum $I_{3}$ vs. ACN mole fraction in water. For description refer to the caption of Fig. 2. 


\section{The models of positron annihilation in liquid mixtures}

The ortho-positronium (o-Ps) pseudoatom can actively interact with non-rigid medium. Assuming that its lifetime depends mainly on feasibility of formation of cavities in liquid structure able to accommodate the electron-positron pair, starting from the Buchikhin et al. [5] model of Ps bubble in a finite potential well, the following relation between the lifetime of the long-lived component of the annihilation spectrum and the surface tension of the medium was suggested by Tao [6]:

$$
\tau_{\text {long,calc }}^{-1}=A \cdot \sigma^{\gamma}
$$

(where $\sigma$ - surface tension, $A, \gamma-$ fitted parameters). This relation is known as the "bubble" model of positron annihilation in liquids. The differences $\Delta \tau_{\text {long }}=\tau_{3 \text {,experim. }}-\tau_{\text {long,calc }}$ are plotted in Fig. 4 against ACN concentration in water. For calculations, the literature data of surface tension of Vierk were applied [7]; the concentration dependence of $\sigma$ is very typical of non-electrolytes in water, with initial rapid decrease and further plateau. In this case the parameters are as follows: $A=1.023 \times 10^{9}, \gamma=0.2984$. It is worth noting that both these parameters have physical meaning resulting from the Tao-Eldrup relation for $o$-Ps lifetime in an infinite well.

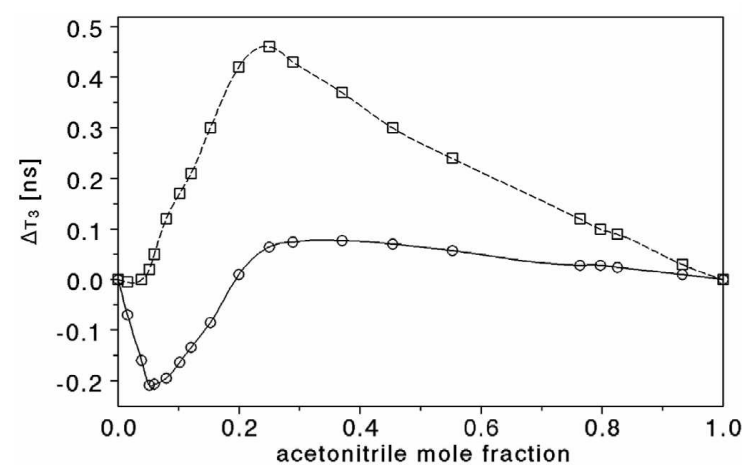

Fig. 4. The differences between experimental and calculated lifetimes of the long-lived component of the annihilation spectrum. Solid line - the model of Tao (Eq. (1)); dashed line - the model of Levay (Eq. (2)). Let us note how different models yield very different plots - most probably they are sensible to different phenomena.

On the other hand, according to Levay et al. [8], starting from geometrical considerations, the lifetime of ortho-positronium in an ideal liquid mixture of liquids $A$ and $B$ can be described as a simple function of concentration

$$
\sqrt{\tau_{3, \text { mixt }}}=V_{\text {mixt }} /\left[\frac{V_{A}}{\sqrt{\tau_{3, A}}}-\left(\frac{V_{A}}{\sqrt{\tau_{3, A}}}-\frac{V_{B}}{\sqrt{\tau_{3, B}}}\right) x_{B}\right],
$$

(where $V_{i}$ - the molar volume of the $i$-th component, $x_{i}$ - its molar frac- 
tion in the mixture). This formula was exploited very often in interpretation of the annihilation data of aqueous mixtures; a good example is our work on water+tetrahydrofuran system [9].

\section{Interpretation and conclusions}

The isentropic compressibility isotherms shown in Fig. 1 intersect at concentrations close to ACN mole fraction of 0.05 . This value suggests that there is an analogy with aqueous ethanol solutions $\left(x_{\mathrm{ACN}}=0.055\right)[3]$, but there are at least two observations that contradict it. Firstly, the intersection concentration is not temperature-independent, shifting quite fast from $x_{\mathrm{ACN}}=0.047$ for $303 \mathrm{~K}$ to $x_{\mathrm{ACN}}=0.055$ for $288 \mathrm{~K}$. Secondly, the compressibility value at the intersection is ca. $4.27 \times 10^{-10} \mathrm{~m}^{2} \mathrm{~N}^{-1}$, the number significantly bigger than compressibility of solid aqueous clathrates determined by Stackelberg and Müller [10]. According to the theory of Endo [11], in aqueous solutions of solutes able to form clathrate-like structures in water and at concentration corresponding to the stoichiometry of the clathrate hydrates, these structures dominate and the compressibility of solution should be very close to that of solid clathrates. This was well proved in many systems, and also for water-ethanol mixtures [3]. Both for ethanol and ACN in water solvent, the clathrates formed should be those known as "structure II" clathrates, with $136 \mathrm{H}_{2} \mathrm{O}$ molecules forming 24 cavities, 8 of them large enough to accommodate molecules of guest-like ethanol or acetonitrile and 16 smaller, which are supposed to contain "monomeric" $\mathrm{H}_{2} \mathrm{O}$ molecules (i.e. not hydrogen-bonded with the infinite network) [12]. In fact, molecules of ACN are smaller than those of ethanol or any other solute forming clathrates of the second type; one could suppose rather formation of the type I clathrates, if any. The molecule of ACN is very similar to that of methanol, which undoubtedly forms clathrates of the first type, with approximate stoichiometry of $\mathrm{X} \cdot 8 \mathrm{H}_{2} \mathrm{O}$ [13]. On the other hand, the experimental results are much closer to those for ethanol (see Figs. 2 and 3 ).

It is possible that $\mathrm{ACN}$ forms clathrate hydrates similar to those of methanol (structure I) but weaker - the idea similar to the interpretation of Benson et al. [14]. This means that increasing concentration of clathrate hydrates reduces their stability and, in concentrations higher than 0.05 ACN mole fraction, the system becomes a mixture of different hydrates, not only clathrate-like ones. Inspection of Figs. 2 and 3 suggests strongly that the title system is closer to water+ethanol than to water+methanol. The most striking analogies are seen in Fig. 2. The only (but substantial) difference is that for both one can distinguish two regions of the dependence of $\tau_{3}$ vs. ACN mole fraction (seen as two almost linear changes for ACN), while only for ethanol there are subtle phenomena in low alcohol concentration, seen as inflection at ethanol mole fraction of 0.05 . In our opinion, the region of rapid increase in lifetime corresponds to structural rearrangements, leading to formation of local crystalline-like structures. If clathrates are formed, the rate of the increase in $\tau_{3}$ becomes slower, resulting in the inflection. 
In the light of above, the final conclusion should be as follows: acetonitrile is too weak hydrophobic agent to stabilize typical clathrate hydrates (type I or type II). It is a weak structure-breaker, more resembling ethanol than methanol and forms with water solvates of untypical, open-work architecture and rather low stability. This is, however, sufficient to stabilize ortho-positronium pseudoatoms in the cavities of such structures and finally one observes the experimental picture of annihilation very similar to that for ethanol.

\section{Acknowledgments}

This work was partially supported by the internal grant of the University of Wrocław no. 2016/GW/IFD/2004.

\section{References}

[1] E. Matteoli, L. Lepori, J. Chem. Phys. 80, 2856 (1984).

[2] A. Wakisaka, H. Abdoul-Carime, Y. Yamamoto, Y. Kiyozumi, J. Chem. Soc. Faraday Trans. 94, 369 (1998).

[3] K. Jerie, A. Baranowski, S. Ernst, J. Glinski, Acta Phys. Pol. A 69, 81 (1986).

[4] See as an example: K. Jerie, A. Baranowski, B. Rozenfeld, B. Jeżowska-Trzebiatowska, J. Gliński, Acta Phys. Pol. A 82, 183 (1992) and our papers cited therein.

[5] A.P. Buchikhin, V.J. Goldanskii, A.O. Tatur, V.P. Shantarovich, Zh. Eksp. Teor. Fiz. 60, 1136 (1971); Sov. Phys. JETP 33, 615 (1971).

[6] S.J. Tao, J. Chem. Phys. 56, 5499 (1972).

[7] A.-L. Vierk, Z. Anorg. Chem. 261, 283 (1950).

[8] B. Levay, A. Vertes, P. Hautojärvi, J. Phys. Chem. 77, 2229 (1973).

[9] K. Jerie, A. Baranowski, B. Rozenfeld, S. Ernst, J. Gliński, Acta Phys. Pol. A 64, 77 (1983).

[10] M. Stackelberg, H. Müller, Z. Elektrochem. 58, 25 (1954).

[11] H. Endo, Bull. Chem. Soc. Japan 16, 1586 (1973).

[12] H. Müller, M. Stackelberg, Naturwissenschaften 39, 20 (1952).

[13] K. Jerie, A. Baranowski, B. Rozenfeld, S. Ernst, B. Jeżowska-Trzebiatowska, J. Gliński, Acta Phys. Pol. A 66, 167 (1984).

[14] G.C. Benson, P.J. D'Arcy, Y.P. Handa, Thermochim. Acta 46, 295 (1981). 